\title{
Identifying student resources in reasoning about entropy and the approach to thermal equilibrium
}

\author{
Michael Loverude \\ Department of Physics and Catalyst Center, California State University Fullerton, \\ Fullerton, California 92834, USA
}

(Received 30 September 2014; published 23 September 2015)

\begin{abstract}
[This paper is part of the Focused Collection on Upper Division Physics Courses.] As part of an ongoing project to examine student learning in upper-division courses in thermal and statistical physics, we have examined student reasoning about entropy and the second law of thermodynamics. We have examined reasoning in terms of heat transfer, entropy maximization, and statistical treatments of multiplicity and probability. In this paper, we describe student responses in interviews focused on the approach of macroscopic systems to thermal equilibrium. Our data suggest that students do not use a single simple model of entropy, but rather use a variety of conceptual resources. Individual students frequently shifted between resources, in some cases leading to contradictory predictions. Among the resources that students employed were some that have been previously described in the literature, including inappropriate use of conservation. However, our results suggest that student use of resources connected to disorder are neither simple nor monolithic. For example, many students used a previously unreported association between the equilibrium state of a system and an increase in order, rather than disorder.
\end{abstract}

DOI: 10.1103/PhysRevSTPER.11.020118

PACS numbers: 01.40.Fk, 05.90.+m

\section{INTRODUCTION}

Entropy is a fundamental concept in the physical sciences and a core idea of thermodynamics and statistical physics. As part of a broader and ongoing project to investigate student learning and develop curricular materials in thermal physics, we have investigated student learning of entropy and the approach to thermal equilibrium.

Entropy is widely known to be a difficult topic for advanced students as well as introductory students. Numerous instructors and authors have suggested methods of teaching entropy, qualitatively and quantitatively, and critiqued existing teaching of the ideas. Until fairly recently, the research base on student understanding of entropy has been fairly limited, and there has furthermore been relatively little in the way of attempts to bridge between the teaching methods and the research.

\section{A. Previous research}

Although entropy is widely considered to be challenging, there is relatively little previous research, particularly for upper-division undergraduates. Prior studies by Duit and Kesidou [1] and Bucy [2] helped to characterize student conceptions of entropy and the second law of thermodynamics; each will be touched upon further in Sec. III C. More recently, several have examined student

Published by the American Physical Society under the terms of the Creative Commons Attribution 3.0 License. Further distribution of this work must maintain attribution to the author $(s)$ and the published article's title, journal citation, and DOI. models of entropy in terms of conceptual metaphors [3]. Cochran and Heron investigated student understanding in the context of heat engines and described several student difficulties with entropy and the second law of thermodynamics [4]. Daane studied the role of energy degradation in courses for preservice teachers focusing on energy models [5]. Additional research in introductory physics courses for life science majors has probed student resources for entropy and spontaneity and has examined similar questions to our study [6]. Probably the most relevant previous work for this paper is that of Christensen et al. [7]. In that study, Christensen et al. reported several common conceptual difficulties with entropy, including a strong tendency of students to conserve entropy inappropriately, and a tendency to assume that the entropy of all systems must increase, whether or not the system is isolated. Another recent study has used tasks adapted from [7] to probe student understanding of entropy [8].

In addition to the systematic study of student learning, many have taken thoughtful positions on the most appropriate ways of teaching entropy. In particular, several critiques have questioned the notion of entropy as a "measure of disorder" as imprecise and potentially misleading [9]. Within the domain of physical chemistry, Lambert has released an influential series of articles of characterizing entropy as disorder, characterizing the notion as a "cracked crutch," and has advocated teaching entropy as a measure of the dispersal of energy in physical space [10]. Further, Lambert criticizes the use of macroscopic examples like a messy dorm room or a shuffled deck of cards. Leff has written extensively on the teaching of 
entropy, including a recent and influential five-part series covering many aspects of the concept and its application in physics [11]. He similarly rejects the idea of entropy as a measure of disorder as "an undesirable simplification of a profound physical entity." He proposes instead that entropy is best thought of in terms either of missing information or of "equity" or "spreading." The latter notion, he suggests, is essentially the same as Lambert's preferred idea of "dispersal."

In recent years, many have proposed a revised instructional approach for upper-division courses on thermal and statistical physics. This approach, often described as "thermal physics" to make a distinction from classical thermodynamics and statistical physics, builds up the second law of thermodynamics as a consequence of the statistical behavior of matter [12]. Several textbooks have adopted this approach, including some for introductory level courses [13] and the text for the courses in this study [14]. We describe this approach in detail in Sec. II A.

\section{B. Theoretical perspectives}

The current study proceeds from the assumption that students construct understanding of scientific phenomena, in some cases developing ideas that are in contrast with accepted scientific viewpoints. The work is primarily empirical and has been directed toward improving student learning in a typical classroom setting, so we started from the pragmatic framework of "investigating student difficulties" [15]. Despite this name, our intention was not simply to identify specific difficulties, but rather to characterize student thinking and reasoning patterns, productive as well as unproductive. However, as the project progressed, it became clear that student responses frequently did not suggest stable conceptual difficulties. Reasoning elements, such as the idea of conservation, could be productive in some contexts and questions but lead to incorrect predictions in others. Individual students, rather than employing a single model repeatedly in a variety of contexts, shifted between ideas frequently. These data suggested the need for a resources, or knowledge-in-pieces, perspective [16].

\section{Research questions}

For this paper we focus on a quite narrow portion of the project. We restrict our focus to student predictions and reasoning in the context of the approach to thermal equilibrium, processes in which objects of different temperature converge to a single equilibrium temperature when placed in thermal contact. In addition, we have chosen to study students not during the thermal physics course in which they first learned this material but a year or more afterward. By performing the interviews well after instruction, we believe we have a sense of the lasting conceptual understanding that remains after students have had the time and opportunity to either integrate their understanding with other courses or forget the material entirely. As a result, the tasks that we have chosen are fairly simple and do not require extensive computation; rather, they focus on the key ideas of thermal equilibrium and the first and second laws of thermodynamics.

The research questions that we have considered include the following:

- To what extent do student responses to entropy questions suggest a consistent and coherent model, and to what extent do responses reflect changing and context-dependent thinking as characterized by a resources model?

- What resources and reasoning patterns do students access in responding to simple conceptual problems involving entropy, the second law of thermodynamics, and the approach to thermal equilibrium?

The goal of this portion of the project is not immediately directed toward the development of curriculum or approaches to teaching, though we might expect that the findings of the work would have implications for instruction. Rather, we seek to characterize how students think about entropy and the approach to thermal equilibrium.

\section{CONTEXT AND METHODS}

\section{A. Instructional context of the work}

The instructional context for this work is a variety of upper-division courses covering thermal physics. The primary context is a thermal physics course at California State University Fullerton (CSUF), a large comprehensive institution in southern California. The course, Physics 310, follows the hybrid thermal physics approach described above, using a popular text [14] that develops the ideas of entropy and the second law of thermodynamics through a statistical approach. The course meets for two 75-minute blocks per week. Enrollments have ranged between 6 and 19 , and typically a significant portion of class time is spent on small-group tutorial exercises, some of which have been described in other publications [17].

Most students in the course are physics majors or minors who have completed introductory physics and several semesters of calculus. The CSUF introductory physics sequence does not include thermodynamics, but many students reported studying thermal physics in high school $(\sim 5 \%)$, in introductory physics courses at other institutions $(\sim 20 \%)$, or in chemistry $(\sim 50 \%)$. A few students $(10 \%-20 \%)$ had previously completed a college-level math course in probability and statistics.

As described above, the course text adopts the hybrid thermal physics approach. This approach seeks to motivate the second law of thermodynamics through an extended logical sequence in which students consider statistical models of phenomena and examine the behavior of these models as the number of particles becomes large. 
Students first consider probability for simple two-state systems (like coins, which can be heads or tails). In this context they consider the key ideas of microstate, macrostate, and multiplicity, and use the fundamental assumption that all accessible microstates of a system are equally probable. The students then determine a means of adapting the expression for multiplicity in the coin system to count states for the Einstein model of a solid. This model is then applied to a system of two interacting Einstein solids. Students are shown that the expected macroscopic outcome (energy shared by the solids in proportion to their respective masses) is the energy arrangement with maximum multiplicity, and thus probability. They then consider larger and larger systems, and apply statistical techniques to show that it is increasingly probable that the system is near the classical equilibrium state. As the number of particles approaches Avogadro's number, the probability of the system being far from that equilibrium state becomes vanishingly small. Thus, if solids are placed together and allowed to exchange energy, they tend to evolve toward a maximum probability state, in which the average energy per particle for each solid is equal. Students are then introduced to the Boltzmann formulation of entropy that relates entropy $(S)$ to the natural logarithm of multiplicity $\Omega$ :

$$
S=k_{B} \ln \Omega .
$$

After this initial statistical introduction, entropy is later introduced as a macroscopic quantity. Students are taught what is sometimes known as the Clausius algorithm, relating macroscopic entropy changes $d S$ to heat transfer:

$$
d S=\frac{d Q_{\mathrm{rev}}}{T} .
$$

They perform qualitative and quantitative analyses of a variety of systems using this algorithm, often involving integration. One touchstone process that students examine is the approach of a pair of macroscopic blocks to thermal equilibrium, in which the heat transfers are equal and opposite but the greater $T$ of the hotter block leads to the smaller absolute value of $\Delta S$. Through this analysis, students are led again to see that the entropy of the Universe must increase in irreversible processes, and connect the macroscopic description of the second law of thermodynamics to the statistical version taught earlier in the course. The students then analyze cyclic processes like those used in engines and refrigerators and use the laws of thermodynamics to derive limits on the performance of those devices.

It is important to note that while the statistical definition of entropy precedes the macroscopic idea and calculations, the two ideas are viewed as equally important. Indeed, the course, text, and instructor spend considerable effort in making connections between macroscopic and statistical pictures. Course assessments include tasks that are both statistical and macroscopic in nature, and indeed students are given exam questions that ask them to transition fluidly between these concepts.

Finally, it is important to disclose that the study author was also the course instructor for the thermal physics course for all students in the study. For this portion of the project, students were asked to participate in interviews only after completing the course, when the instructor would have no further opportunities to assign student grades.

\section{B. Methods}

In the broader project of which this study is a part, we have sought to document student understanding of the target ideas using written conceptual questions and student interviews. In this portion of the study, we describe student responses from a set of interviews. We chose to study the responses of students a year or more after completion of the thermal physics course to probe the longer-term effects of the instructional approach.

We interviewed individual volunteers, most of whom had completed the course 1-2 years prior to the interview. Students were compensated with a gift card. The sample of students included eight students: 4 male and 4 female. All were physics majors, though several had a second major or minor in another discipline. Student ethnic identification was two white, two Asian-American, and four Latino or Latina. The interview participants' course grades were roughly representative of the course as a whole: $1 \mathrm{~A}$, $3 \mathrm{~B}, 4 \mathrm{C}$. In the text below, students are identified with pseudonyms or, in the context of student quotes, with a one- or two-letter abbreviation as indicated in Table I.

TABLE I. Student pseudonyms and abbreviations.

\begin{tabular}{lcc}
\hline \hline Student pseudonym, abbreviation & Demographics & Time since completing course \\
\hline Calliope, C & Female, Latina & $1 \mathrm{yr}$ \\
Darius, D & Male, Latino & $6 \mathrm{yr}$ \\
Falcata, F & Male, Latino & $1 \mathrm{yr}$ \\
Gladius, G & Female, Latina & $2 \mathrm{yr}$ \\
Hecate, H & Female, White & $2 \mathrm{yr}$ \\
Jason, Ja & Male, White & $2 \mathrm{yr}$ \\
Jocasta, Jo & Female, Asian & $2 \mathrm{yr}$ \\
Pericles, P & Male, Asian & $1 \mathrm{yr}$ \\
\hline \hline
\end{tabular}




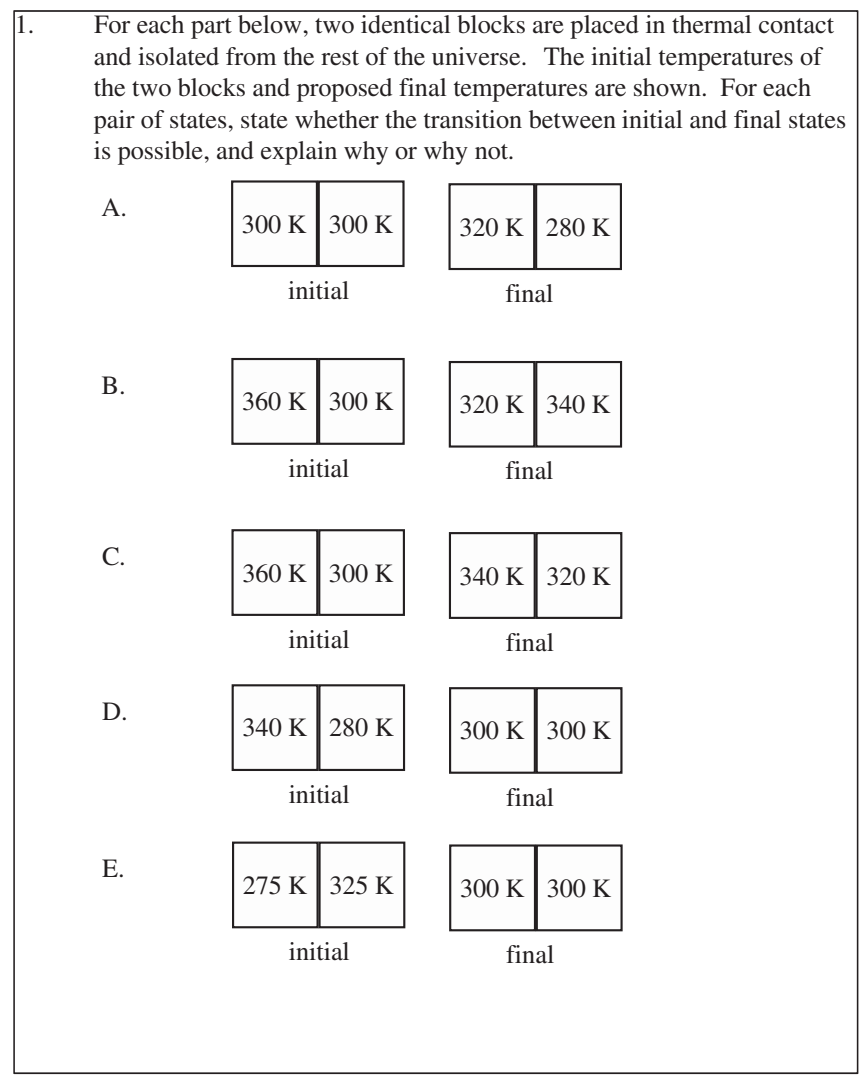

FIG. 1. The first page of the interview task. Students were asked to respond to the questions and explain their thinking. For the first set of four interviews, only three parts (A, D, and E) were used.

In the interviews, students were given a sheet of written questions and asked to answer the questions while thinking aloud. The interviewer followed a protocol that included explicit follow-up questions asked of all students but provided latitude to probe student responses further.

The first set of tasks involved pairs of blocks (see Fig. 1.) Students were given the temperatures of blocks in the initial and final states and asked to identify which of the situations were physically possible and explain why. For the first four interviews, only situations A, D, and E were considered. The other situations were added for the next four interviews at the suggestion of instructors and a second researcher, to investigate how students would think about a system that "overshoots" equilibrium or one that does not fully reach equilibrium. In case $\mathrm{C}$, the intention was that the system would evolve to the final state and stop. Our emphasis in the analysis below is on situations $\mathrm{A}, \mathrm{D}$, and $\mathrm{E}$, which were posed to all students.

The interviewer prompted the students to give explanations but for the most part did not probe these explanations immediately. After students had explained their responses, they were directed to the back of the sheet, which included the "general-context" problem from Christensen et al. [7], shown in Fig. 2.
2. For each of the following questions consider a system under going a naturally occurring ("spontaneous") process. The system can exchange energy with its surroundings.

a) During this process, does the entropy of the system $\left[\mathrm{S}_{\text {system }}\right]$ increase, decrease, remain the same, or is this not determinable with the given information? Explain your answer.

b) During this process, does the entropy of the surroundings [ $\left.\mathrm{S}_{\text {surroundings }}\right]$ increase, decrease, remain the same, or is this not determinable with the given information? Explain your answer.

c) During this process, does the entropy of the system plus the entropy of the surroundings $\left[\mathrm{S}_{\text {system }}+\mathrm{S}_{\text {surroundings }}\right]$ increase, decrease, remain the same, or is this not determinable with the given informa tion? Explain your answer.

FIG. 2 Second page of the interview prompt. Adapted from Christensen et al. [7].

After the students had answered the abstract context question, the interviewer probed the student reasoning. After this discussion, the students were redirected to the front page, and the interviewer asked explicitly whether the students had thought about energy, entropy, and multiplicity or probability, and how those concepts would apply to the problems.

Correct answers to the problems could be arrived at in a number of ways. The simplest formulation is to recognize that the processes must satisfy the first and second laws of thermodynamics. As the blocks are isolated from the rest of the Universe, the energy lost by one block must be gained by the other; as the blocks are identical, their temperature changes should be equal and opposite. All but one of the five processes conserve energy and satisfy the first law. However, one case involves a process that is the reverse of the approach to thermal equilibrium: case A starts with blocks at the same temperature of $300 \mathrm{~K}$ and ends with one block at $320 \mathrm{~K}$ and the other at $280 \mathrm{~K}$. The change in entropy of the left block is positive, that of the right block, negative. However, by considering the Clausius algorithm we can see that the absolute value of the change for the left block is smaller than that of the right block, and the process results in a net decrease in entropy of the two-block system, violating the second law of thermodynamics.

\section{Data analysis}

In analyzing student interview responses, a full transcript was produced. The researchers then coded each student sentence based on its physics content. Rather than focusing on whether responses were correct or incorrect, each sentence was coded as corresponding to one or more common recurring ideas. While this project originally was characterized by a student difficulties perspective, it became clear that student responses were not consistent with students repeatedly employing a single model in their responses. Rather, students shifted between ideas, employing multiple ideas throughout the interview in response to 
TABLE II. Entropy-related resources coded and examples.

\begin{tabular}{|c|c|}
\hline Entropy-related resources & Example student utterance \\
\hline Entropy is a form of energy & I want to say that entropy is a form of energy, but I don't remember exactly. \\
\hline Entropy is conserved & $\begin{array}{l}\text { Entropy you can't just, I can't really remember, but I want to say, it's like } \\
\text { energy, like you can't create it or destroy it. }\end{array}$ \\
\hline Entropy of a system must always increase & $\begin{array}{l}\text { Yes, because even though ... one is increasing in temperature, one is decreasing } \\
\text { in temperature, they are both moving towards the maximum number of } \\
\text { microstates. }\end{array}$ \\
\hline Entropy is a measure of disorder & It's just weird because whenever I think of entropy I just think of more disorder. \\
\hline Entropy is related to multiplicity & $\begin{array}{l}\text { [entropy will] increase in that it has the most possible number of, um, micro or } \\
\text { macrostates. }\end{array}$ \\
\hline Entropy describes dissipation & $\begin{array}{l}\text { Entropy is a more general way of thinking about energy. ... To, um, say a higher } \\
\text { potential of energy wants to dissipate to a lower potential of energy. }\end{array}$ \\
\hline Entropy relates to dispersal & $\begin{array}{l}\text {...like the energy's being dispersed more evenly, and that, would yield an } \\
\text { increase in entropy }\end{array}$ \\
\hline$\Delta S$ must be positive (or $\geq 0$ ) & $\begin{array}{l}\text {...the combination of the two blocks, [entropy] is increasing or remaining the } \\
\text { same }\end{array}$ \\
\hline Entropy is related to enthalpy & it's almost like they're interchangeable, delta $H$ and delta $S$ \\
\hline
\end{tabular}

TABLE III. Equilibrium-related resources coded and examples.

\begin{tabular}{lc}
\hline \hline Equilibrium-related resources & \\
\hline $\begin{array}{l}\text { Systems naturally move "toward" equilibrium rather } \\
\text { than away }\end{array}$ & $\begin{array}{c}\text { Which makes me think this was not possible, just because systems want to } \\
\text { be in a state of more equilibrium. } \\
\text { Equilibrium decreases multiplicity } \\
\text { Temperatures going away from thermal equilibrium, I'm just thinking of } \\
\text { that as decreasing multiplicity. }\end{array}$ \\
$\begin{array}{l}\text { Application of energy can oppose equilibrium } \\
\text { it takes energy to keep it that way [away from equilibrium], then it } \\
\text { naturally goes back, spontaneously }\end{array}$ \\
$\begin{array}{l}\text { it's going from a state of disorder to order, to some sort of order, so that's } \\
\text { why I would assume, there's some kind of order in equilibrium }\end{array}$ \\
$\begin{array}{l}\text { Equilibrium maximizes multiplicity or probability } \\
\text { occur. And it will always move towards that central peak. }\end{array}$ \\
\hline \hline
\end{tabular}

different prompts. In many cases the ideas were conflicting and even contradictory, even within a single response. Students frequently recognized that they had contradicted themselves and struggled to resolve the contradiction. Thus, our analysis focused on the characterization of student ideas using smaller elements of knowledge.

The appropriate grain size of these smaller knowledge elements bears some discussion. The ideas we have documented are typically more than diSessa's $p$-prims [18], as they are connected to specific physics concepts and frequently span multiple sentences. These ideas might best be described as facets, as they were not complete concepts but rather partial concepts that were more descriptive of aspects of a situation or problem [19]. In any event, our goal is not to make particular claims about the appropriate grain size, and in the subsequent discussion we refer to these student ideas using the somewhat agnostic term "resource" as used by Hammer [16]. In Tables II and III we give examples of the resources that we identified and examples of student utterances that exemplify these resources. Some of these resembled canonically "correct" physics ideas ("entropy is related to multiplicity"), but others did not ("entropy is a form of energy"). The ideas as coded are broken roughly into resources related to entropy and those related to thermal equilibrium, though there are examples of utterances that cross this boundary. Given the limited data set, we do not claim this is a comprehensive set of student resources, but it does represent the resources coded within this interview sample.

\section{CHARACTERIZATION OF STUDENT RESPONSES}

\section{A. Entropy and multiplicity}

In this sample, the connection between entropy and multiplicity was not strong for most students. Two of the students expressed strong preferences for multiplicity over other ways of thinking about entropy. However, one of these students, who used microstates to anchor her prediction about entropy, acknowledged that she often could not apply the idea of multiplicity or distinguish between microstates and macrostates. (In a related article, we 
describe in more detail student reasoning difficulties with the concepts of multiplicity, microstate, and macrostate [20].) The other students in the sample expressed either discomfort with this connection or outright confusion. Many of the students cited $S=k \ln \Omega$, but the majority of students did not articulate strong connections between multiplicity and the equilibrium-seeking behavior they were describing. For example, Jocasta seemed to connect multiplicity (and probability) with a notion of plausibility, rather than a quantity describing microstate:

Jo: Not really, um, multiplicity the way I remember it, it's like can it happen? It's like flipping a coin, each time it happens, it's random, it's not influenced by the other time it's flipped. I didn't really think about that.

On the other hand, there was one student in the sample for whom the statistical idea of entropy as being connected to multiplicity was very useful:

Ja: I'd never heard entropy described in a multiplicity sense, like a statistical sense. It made so much more sense, because every time you hear disorder, and I hear that from people who, like, don't know anything about physics: "It's just disorder."... But I don't think they know what that means. I never knew what that meant.

His unhappiness with the idea of order may have related to an issue described further in Sec. III C 2, as he stated that he associated the equilibrium state with more order (or, at least, with "more orderly") rather than more disorder:

Ja: I feel like it [equilibrium state] would be more orderly, if anything. ... Because, it feels like everything's moving to the most likely position because, that's just how it is.

While it was a key part of the arguments given by Hecate and Jason, the formulation of entropy as a statistical quantity was not productive for most of the students. That is particularly notable given the fact that Boltzmann formulation of statistical entropy was a major focus of the course and the instructional approach.

\section{B. Macroscopic entropy}

One key physics idea was notable for its absence in student responses: none of the students gave responses to the entropy changes using the macroscopic idea that

$$
d S=\frac{d Q_{\text {rev }}}{T}
$$

(sometimes referred to as the Clausius algorithm). It is possible that the lack of references to this idea reflects the emphasis of the thermal physics approach, in which entropy and the second law of thermodynamics are introduced in terms of statistical behavior. Without studies of students in a traditional thermodynamics course, in which entropy is first introduced macroscopically, it is impossible to say whether this is particular to the thermal physics approach or a characteristic of students thinking more broadly. Although many of the tasks in the interview could be answered without referring to the Clausius algorithm, the macroscopic analysis of the two-blocks tasks in terms of the second law of thermodynamics requires this step.

\section{Difficulty relating entropy changes to temperature changes}

After students had completed the abstract-context task on the back page, they were asked to describe the usefulness of entropy in thinking about the pairs of blocks on the first page. In most cases, the interviewer chose one of the situations and asked specifically what would happen to the entropy of each block and to the entropy of the twoblock system. This task was simple for some students, but others struggled. Even students with a largely sound understanding did not typically reason in terms of heat transfers and temperatures. Hecate mistakenly predicted that both blocks in part A would decrease in entropy, without referring to the heat transfers:

$\mathrm{H}$ : That would mean that the entropy of both blocks in A would be decreasing, because they are moving away from the stable equilibrium state.

Jason, who made many correct predictions and articulated the connection between entropy and multiplicity, struggled when asked about the entropy changes of individual blocks:

I: What can you say about the entropy of the left block and the right block?

Ja: The left block is increasing its entropy.

I: How about the right block.

Ja: I think it's decreasing...?

I: But you're not sure?

Ja: But I'm not sure. [laughs]

I: How come?

Ja: I'm trying to remember how, um, entropy is related with temperature. So I'm not really, um, sure about that.

The interviewer tried a second task, and asked Jason about the changes in entropies of the two blocks in part B:

I: If I thought about the entropy of the two pieces there, what could I say?

Ja: Individually? [laughs] I don't really know individually. But as a whole, I would say the entropy is decreased. But individually, I think, that's kind of wracking my brain right now.

Later Jason expressed confusion when asked to compare macroscopic and statistical models, and explicitly stated that the probabilistic interpretation was vastly preferable to him: 
Ja: every time somebody asked me for the definition, I always think about it in that probabilistic or multiplicity kind of way. [Darius] was doing his chemistry homework and I was looking at it that way.

The fact that the two students most aligned with a statistical approach had difficulty with a very simple task involving macroscopic entropy is certain cause for reflection and further research. Whether this is idiosyncratic or reflects a deeper conflict between statistical and macroscopic pictures of energy remains an unanswered question.

\section{Entropy must increase}

A key finding of Christensen's work was that students tended to predict that the entropy of systems must increase, regardless of whether the system in question was isolated. In our study this tendency was also present, but did not appear to be as widely applied. All eight of the students at some point stated explicitly that entropy of some system would increase. In three of the eight cases this was stated carefully, as a physicist might state the second law of thermodynamics: the change in entropy of the Universe must be greater than or equal to zero. Other students spoke more generally about the tendency of entropy to increase, without specifying which system(s) were being described. Three of the eight students used conservation reasoning for entropy at one point while also stating that the entropy of an individual (not necessarily isolated) system must increase. The remaining two students primarily used conservation reasoning.

\section{Conservation of entropy}

As has been reported in previous studies, several student responses were consistent with the idea that entropy is a conserved quantity. In some cases, students explicitly related entropy to energy. Pericles, in responding to the prompt, stated that, "I want to say that entropy is a form of energy, but I don't remember exactly." After the interview he suggested that he had thought that entropy was another one of the thermodynamic potentials that are taught in the course [21]. Other students described entropy as having units of Joules, though in at least one case the association was with enthalpy rather than entropy:

I: So this, the $5 \mathrm{~J}$, is this the entropy you're talking about?

D: That's just the, that's the change in uh, that's delta H. But. The reason why I say it's the entropy, cause I think somewhere we said in our chemistry class, it's almost like they're interchangeable, delta $\mathrm{H}$ and delta S. ... I'm struggling to remember. I think they were interchangeable. According to them. The chemists.

Another student, Calliope, was particularly explicit in making the connection to energy and invoking conservation:
C: You can't just, entropy ... I can't really remember, but I want to say, it's like energy, like you can't create it or destroy it, it can't just come out of nowhere.

In the context of an energy discussion, she spoke more generally about the value of conservation, suggesting that she felt it was very broadly applicable:

$\mathrm{C}:[\ldots]$ Things have to be conserved. That's like the main thing they teach us, since like middle school or high school. Like, things just don't appear out of nowhere, they have to come from somewhere.

In response to the Christensen task, Jocasta used very explicit conservation reasoning and appeared to equate energy and entropy:

I: You said the entropy of the left box would go up, and the entropy of the right box would go down, then you said something about the total being, or the average, being the same.

Jo: The sum of them would be the same.

I: And how do you know that would be the case?

Jo: [quickly] conservation of energy.

\section{Entropy as a description of dispersal}

As noted above, several have recently advocated teaching entropy in terms of ideas like spreading or dispersal. The textbook, lectures, and curricular materials in this course did not explicitly introduce the idea of entropy using either approach, but a few of the students employed resources of this nature nevertheless. For example, Jason in rejecting the notion of entropy as disorder explicitly used the phrase "dispersed more evenly" to describe the approach to equilibrium.

Ja: The way I think about entropy is more the statistical sense, we always talked about a measure of disorder, but that's sort of inaccurate, it's more like it's more orderly in the sense that everything's fitting into its corresponding slot, like the energy's being dispersed more evenly, and that, would yield an increase in entropy.

Another student, Pericles, used what might be a similar notion, that of dissipation. Based on the way Pericles describes it, dissipation seems to mean the same as dispersal, i.e., spreading through space.

P: Entropy is a more general way of thinking about energy. But it's more broad in a sense. Because you have exchange of energy, you have more energy that wants to dissipate. To, um, say a higher potential of energy wants to dissipate to a lower potential of energy.

However, Pericles did also use what we coded as a conservation resource when talking about entropy, and (as noted above) described entropy as a "form of energy." In 
the Christensen task he predicted that the total entropy of system plus surroundings must remain the same.

While our small sample does not permit judgment that this model is more or less useful than any other, it does suggest that for some students at least the notion of a quantitative measure of dispersal is a resource upon which instructors might build. However, some caution should be applied, as most of the students did not refer to dispersal or spreading when describing their thinking about entropy, and one of those that did apparently still thought of entropy as a conserved quantity. Even in Jason's quote, the dispersal resource is used in connection with a discussion of whether a system is "orderly."

\section{Entropy and disorder}

As noted above, the characterization of entropy as a measure of disorder is a controversial one. Nevertheless, the association of entropy with order and disorder is seemingly inescapable. Of the students in this sample, only one did not introduce the idea of disorder when speaking about entropy. Two of the students who spoke about disorder did so primarily as a disclaimer, stating that they preferred other ways of thinking about entropy. The remainder of the students used disorder frequently and spontaneously.

Many have criticized teaching entropy as a measure of disorder, but research on the question is limited. Kesidou and Duit [1] note that students use this notion readily, but they claim that students have insufficient particulate understanding to fully understand it. Bucy [2] came to a similar conclusion and noted that students use the term in disparate ways.

\section{Entropy as disorder is a more subtle idea than one might think}

While the association of entropy with order and disorder was widespread, even within this fairly small sample, the use of disorder took a number of forms. In their responses, students used a variety of phrasings to describe systems and their level of order or disorder. Their phrasing suggested that in some cases order was a quantity, but in others it was a description of a state, or even a state itself:

disorder is a little more natural that would be disorder

you would be changing it toward disorder

the system is getting less ordered

it would be more disordered

it's still disordered

equilibrium state is more disorder

from a state of disorder to order

it would go toward the most disorderly state

A fairly dramatic example comes from the responses of Gladius. In her analysis, Gladius frequently referred to disorder. However, she used the term in at least two different ways. Early on, she seemed to refer to disorder as a state, that a system could be in disorder, rather than a semiquantitative construct that might increase or decrease:

G: You can't have this go below this; That would be disorder.

G: I don't think that would happen, just because it's disorder.

Later, her language shifts subtly, to refer to "more disorder," suggesting disorder as a quantity rather than a state. Note that her usage is a hybrid: the final state is more disorder rather than has more disorder or is more disordered.

\section{$\mathrm{G}$ : The final state is more disorder.}

As the interview progresses, she shifts to a more standard usage; this may be in part due to inadvertent cuing from the interviewer:

G: Because I said this one would be more disordered, so this one should have more entropy.

Calliope frequently invoked the macroscopic notion of "messiness" as a proxy for entropy, but did not speak of more or less disorder. Rather, she referred to a room as disordered, and referred to an association of entropy and order or disorder, but in a way that suggests order or disorder as states rather than measurable quantities:

...disorder is a little more natural.

If we say that disorder goes with entropy...

... unless entropy comes with order.

....if entropy comes with disorder...

Falcata used the idea of disorder and order extensively. While he generally spoke of a state being "more disordered" or "less orderly," he also appeared to use terms in a manner similar to Gladius:

$\mathrm{F}$ : The equilibrium state is more disorder...

F: If it started off in its equilibrium state, that would be its ordered state.

\section{Some students have unexpected associations of entropy and order}

In our study, there was evidence to suggest a previously unreported association with entropy. Four of the eight students in this sample gave responses suggesting an association of the equilibrium state of a system with greater order rather than disorder, and in some cases greater entropy.

One student (Jason) seemed to reject the relationship of entropy to disorder based on the notion that an equilibrium state seemed more orderly:

Ja: I think the system has to be increasing in entropy. The way I think about entropy is more the statistical sense, we always talked about a measure of disorder, but that's sort of inaccurate. It's more like it's more orderly in the sense that everything's fitting into its corresponding slot. 
Upon probing by the interviewer, he went on to confirm that he intended to say that the equilibrium state would be more "orderly":

Ja: I feel like it would be more orderly, if anything. And not just because you said that! Because, it feels like everything's moving to the most likely position because, that's just how it is.

The statement "not just because you said that" gave the interviewer pause; it was not clear whether this was a reference to a statement earlier in the interview or a statement in class. To be certain of the student's intent, the interviewer probed further, and the student emphatically agreed:

I: And correct me if I am wrong, because I don't want to put words in your mouth, but it sounds like you're saying that the most probable state seems more orderly to you, as opposed to more disorderly.

Ja: [student interjects] Yes!

I: Is that what you mean?

Ja: Yes, yes. Cuz that's the way the world kind of works, stuff tends to follow probability, that's why they are probabilities.

This student seemed to have integrated the relationship between entropy, equilibrium, and order into a relatively correct understanding by rejecting the association of entropy with disorder. Other students did not resolve this confusion in the same way. When asked whether entropy would help to understand which two-block processes were possible, Jocasta said:

Jo: It does help, seeing that it went from more entropy to less entropy... in a state of more equilibrium.

Note that an accepted explanation of the evolution of a system to thermal equilibrium is that the entropy is greater in the final state. This student seems to say otherwise, and a follow-up question both confirmed this and revealed confusion between entropy, order, and equilibrium.

Jo: It's just weird because whenever I think of entropy I just think of more disorder, and so whenever I look at this, it's going from a state of disorder to order, to some sort of order, so that's why I would assume, there's some kind of order in equilibrium.

Another student, Calliope, brought up the idea of entropy and her messy room, but seemed uncertain whether to associate entropy with disorder or with order:

$\mathrm{C}$ : If entropy relates to disorder, then I guess a disordered... If we say that disorder goes with entropy, then my room would have more entropy, unless entropy comes with order. Hm.

These student responses seem to indicate that there is a conflict for some students between the association of entropy and disorder and the association of the equilibrium state with higher disorder and (or) entropy:
C: It doesn't sound right, it doesn't sound right, that [the blocks would] be at a high-entropy state, when they're in their natural state.

A fourth student stated that the initial state of two blocks with different temperatures (case E from Fig. 1) would have more disorder than the state in which the blocks have reached thermal equilibrium:

G: I think this one has more disorder. The initial state. Because it's not happy, it's not where it wants to be. Whereas, the final state, you know, everything is equal, it's kind of like a, like a stable, environment.

A similar association of equilibrium with order is reported in the recent study by Geller.

\section{Avoiding "entropy as disorder" does not prevent students from employing the resource}

The course in the study did not teach entropy in terms of disorder, and the instructor (and study author) indeed avoided the term entirely except when cautioning students that disorder can be imprecise, perhaps even misleading and ambiguous. The course textbook similarly avoided entropy as disorder, and gave an example intended to illustrate its ambiguity. Despite these efforts, nearly all of the students in the interviews referred to disorder at some point during the interviews. Only one student did not mention disorder; two of the eight mentioned it but primarily to state their dislike of the idea. For the other five students, "entropy is a measure of disorder" was a resource employed at least occasionally in solving the interview tasks. It is clear that actively avoiding the term disorder and cautioning students about it does not prevent them from reasoning with it. The recent work by Geller [6] also concludes that the prior experience of students with notions of disorder is nearly inescapable.

At the same time, many of the students who used entropy as disorder extensively came to a point in the interview when they recognized some ambiguity in their usage. Several of these examples came up in the context of the association of order with equilibrium, above:

C: If we say that disorder goes with entropy, then my room would have more entropy, unless entropy comes with order. Hm.

In addition to the examples from the previous section, Falcata recognized the ambiguity in his usage:

F: So, what I'm thinking now is that, well, ordered, um, I'm using it very vaguely. And it would be helpful if I'd kind of stick to something, um. I am thinking back to an example of marbles in a box, shaking them up, if they were organized by color, or had numbers on them or something.

F: When I meant that that was its more ordered state, I meant was, if you changed it. You would be changing it toward disorder. So I guess I wasn't really clear on what I meant by order and disorder. 


\section{CONSISTENCY OF STUDENT RESPONSES}

Previous reports on student understanding of entropy have characterized student responses in terms of common incorrect answers. Although there were a few strong ideas that were used by multiple students, perhaps the most striking feature of student responses was the lack of consistency. Of the students in the sample, most did not repeatedly and consistently employ a small set of the resources described above. In contrast, we found in our analysis that students in giving explanations would shift between ideas similar to previously reported common incorrect answers and canonically correct physics ideas. For example, a student might in one statement articulate the idea that entropy must increase, and then later state that entropy cannot be created nor destroyed. In the discussion below, we give three somewhat extended examples of student responses that illustrate the extent to which students shifted among the resources described above.

\section{A. Example 1: Calliope}

Calliope's responses throughout the interview were particularly striking, as she made repeated use of several distinct, and often contradictory, resources in constructing her responses to the questions. Her explanations bounced between conservation, entropy as disorder, equilibrium is ordered, and the idea that equilibrium (and entropy) opposes energy.

Unlike many students, Calliope introduced the idea of entropy early on, though she struggled to remember whether the idea she was trying to express was associated with the term entropy or enthalpy. (She had completed the second semester of general chemistry just before the interview and the thermal physics course a year earlier, which may help to explain this phenomenon.) She invoked a common metaphor for entropy, the "messy room."

C: I can't remember the difference between entropy and enthalpy. I have to look through the definition. Was entropy where everything was, wanted to go back to where it should be?

I just remember this, I don't know if we talked about it in this class, just explaining to your mom why your room is messy, and you can say it's because of a word from thermo, but is it entropy or enthalpy?

In response to the Christensen questions, Calliope gave explanations consistent with the idea that entropy is a conserved quantity.

$\mathrm{C}$ : This one [sum of system and surroundings] remains the same. And I feel like if this one increases, if the system increases, then the surroundings decrease, and if the system decreases then the system, it's like the opposite. If one increases the other decreases.

This response is highly suggestive of conservation reasoning, and indeed Calliope later explicitly related the behavior of entropy to that of energy, and was very explicit in invoking conservation:

C: You can't just, entropy you can't just, I can't really remember, but I want to say, it's like energy, like you can't create it or destroy it, it can't just come out of nowhere.

From this work as well as other studies in the thermal physics project, we believe that for physics majors in particular the idea of conservation is a strong one. When confronted with new ideas like entropy or multiplicity, students frequently employ a conservation resource. Calliope talked about energy conservation later, but her language suggested a very general approach to conservation:

C: Things have to be conserved. That's like the main thing they teach us, since like middle school or high school. Like, things just don't appear out of nowhere, they have to come from somewhere.

In responding to the Christensen questions, Calliope first answered that the entropy of the system in part A must increase, but then quickly changed her mind to say it decreased. She struggled a bit with the abstract nature of the task and returned to her example of the messy room:

C: When I think about these things, I think about them in a situation. I need to picture something. So I think about my room, clean, its natural state is dirty, so would it increase or decrease....

However, while she was certain that the natural state of her room was to be messy, she was unsure whether this messy state corresponded to more or less entropy.

I: Does that correspond to more entropy or less entropy, when your room is messy?

C: [Pause] I think less. I guess I'm relating entropy to energy, I wanna, just really want to put those two together. I'm sure they do go together, but I don't really remember exactly how.

However, she also seemed to make connections between entropy and energy that suggested that she thought of them as opposing equilibrium and (or) one another, which may again be related to her recent experience in a general chemistry course:

C: Well if we think about, if I think about a gas, and its kind of forced to be a way, and it takes energy to keep it that way, then it naturally goes back, spontaneously, so I'm gonna say it [entropy] decreased. There was some sort of energy to make it be there in the first place.

Calliope did not introduce the idea of order or disorder, but the interviewer prompted her to clarify whether her descriptions of the messy room were related to notions of disorder. She associated disorder with the "natural" state of a system. However, her connection between order or 
disorder and equilibrium shifted between conflicting resources. By "natural" she appeared to be referring to an equilibrium state, a relaxed state of a system. For a moment she stated that the natural state was the more disordered state, in agreement with the widespread (albeit controversial) notion of entropy as disorder as macroscopic disorganization.

C: To me, disorder is a little more natural. My room just doesn't want to stay clean. [laughs] It takes more work to keep it in a certain state that it doesn't really want to be in.

The interviewer sensed that this answer may have contradicted previous responses on entropy, and prompted her to reconcile them. Calliope recognized the contradiction and struggled to reconcile her conflicting intuitions, going back and forth several times:

I: In the example of your room...earlier you said the messy room had less entropy, is that correct?

C: Hm. Hm. I just, [pauses] I guess if I think about it that way, it would...I'm not really making sense, because if I'm saying, entropy, or [pause] I, If entropy relates to disorder, then I guess a disordered... if we say that disorder goes with entropy, then my room would have more entropy, unless entropy comes with order. $\mathrm{Hm}$.

She continued to attempt to reconcile the notions of entropy, disorder, and equilibrium, using both canonical and noncanonical ideas. She changed her mind and stated that her intuition suggested the following:

C: Now I want to say it's increasing. Before I was almost certain that it was decreasing. And now that we're saying this, if we relate entropy to the state of being messy, then it would be higher.

Rather than describing equilibrium, she continued referring to the natural state of a system:

C: Well, if entropy comes with disorder, and it goes to its more natural state, then my room's entropy would increase, the messier it would get.

I: So your natural state, or a system's natural state, is that a high-entropy state or a low-entropy state?

C: Hm. [pause] I want to say, low. No! Well, if they're inversely proportional, then yes. ...

I: The natural state is, a high-entropy state?

C: It doesn't sound right, it doesn't sound right, that they'd be at a high-entropy state, when they're in their natural state.

She switched back and forth several more times:

C: I can't just apply every thing to my room, because there are things that like in the natural state, they're like, the chairs are stacked together, and they're right the way they are, it doesn't take any energy or whatever. Geez, I'm just confusing myself.
I want to say when, like it doesn't take any energy to keep it, but if they're inversely proportional it would be at a high entropy. No that doesn't sound right. I'm gonna go with low.

In the end, Calliope decided that the equilibrium state would correspond to low entropy, adding, "You know when I get out of here I'm going to look that up, right?"

\section{B. Example 2: Gladius}

Another student, Gladius, displayed a similar shift between ideas. In response to the abstract-context question, she gave a response that seemed to be consistent with the second law of thermodynamics. However, her response does state that the entropy of a system must increase, rather than the entropy of the Universe, as previously identified by Christensen:

G: If it's spontaneous, I think, for the first one, the entropy of the system, it's naturally occurring, I think [faintly]. I want to say it's increasing.

In her analysis, Gladius frequently referred to disorder. However, she used the term in different ways during the course of the interview. At one point she seemed to refer to disorder as a state, that a system could be in disorder, rather than a semiquantitative construct that might increase or decrease:

G: You can't have this go below this; That would be disorder.

G: I don't think that would happen just because it's disorder.

Later, her language shifts subtly, to refer to "more disorder," suggesting disorder as a quantity rather than a state. Note that her usage is a hybrid: the final state is more disorder rather than has more disorder.

G: The final state is more disorder.

As the interview progresses, she shifts to a more standard usage; this may be in part due to inadvertent cuing from the interviewer (see the question posed below). In her discussion of part $\mathrm{E}$ of the two-blocks question, she shifted between three ideas. First, she gave the quote from the previous section, indicating that the different temperatures of the initial state corresponded to more disorder:

I: Which has more disorder overall?

G: I think this one has more disorder. The initial state. Because it's not happy, it's not where it wants to be. Whereas, the final state, you know, everything is equal, it's kind of like a, like a stable, environment.

Then, she said the initial and final states have the same entropy, seemingly a conservation argument:

I: Which situation would you say has more entropy, the initial or the final?

G: I want to say that they both have the same. Yeah. 
After the interview probed further, she gave an answer suggesting the interpretation of entropy as a measure of disorder, increasing as a system approaches equilibrium:

I: Is that consistent with what you said about order or disorder? Or should it be?

G: No it's not.

Because I said this one would be more disordered, so this one should have more entropy. But I wouldn't know how to describe that.

Note that in very short order she has used three ideas that a physicist would identify as distinct, and contradictory, with potentially three different conclusions about the change in entropy of the system. But she seemed to settle into the notion that entropy would decrease as the system approached equilibrium:

G: Entropy would kind of equal out, that's why I was able to say, it would come to some kind of equilibrium. It would go from a higher entropy to a lower entropy.

G: ...but entropy does help, seeing that it went from, more entropy to less entropy, in a state of more equilibrium.

The interviewer asked again to clarify whether the equilibrium state would correspond to more or less entropy:

I: so is 'more equilibrium' associated with less entropy?

G: Yes? [laughs nervously]

G: It's just weird because whenever I think of entropy I just think of more disorder, and so whenever I look at this, it's going from a state of disorder to order, to some sort of order, so that's why I would assume, there's some kind of order in equilibrium.

In this case equilibrium is also a hybrid usage; "more equilibrium" is suggestive of a continuous quantity rather than a yes or no state. Such a usage may simply reflect a shortening of the phrasing "closer to" or "further from" equilibrium.

These two statements seem to be in conflict, but one refers to disorder and one to entropy, so it is not clear whether it represents a contradiction or a difficulty in relating entropy to order or disorder. Therefore, a few moments later, the interviewer probed for consistency, and Gladius changed again to relate entropy to disorder, saying:

G: I said this one would be more disordered, so this one should have more entropy. But I wouldn't know how to describe that.

Ultimately, she was unable to reconcile the confusion.

\section{Example 3: Hecate}

Hecate stands in sharp contrast to most of the students in the sample, as she did not choose to describe entropy in terms of disorder, and indeed explicitly rejected the notion. She preferred a statistical interpretation, though she acknowledged that this might be inaccessible for introductory students:

H: Disorder is generally a good way to introduce the concept at a lower level.

I'm not gonna lie, S equals natural log of the states, using a mathematical relationship with probability, is not a good first introduction.

Disorder is an easy thing to, it's something to point us to.

Despite her stated preference for statistics, Hecate seemed to struggle at times to reconcile statistical measures of a system with the macroscopic questions about entropy and the approach to equilibrium. Hecate sketched a graph of multiplicity that was referred to frequently in class, and correctly noted that the process would spontaneously evolve toward the central peak, which corresponds to the classical equilibrium state as well as a maximum of the multiplicity of the combined system. However, she could not decide whether this spontaneous evolution toward equilibrium was consistent with an increase or decrease in entropy:

$\mathrm{H}$ : And it will always move towards that central peak. Never away from it, as a natural spontaneous process. What I don't really remember is whether that corresponds to an increase in entropy or a decrease, just in terms of terminology.

When prompted by the interviewer what her intuition suggested, she promptly responded that her intuition was that the entropy would increase:

I: What's your tummy tell you?

$\mathrm{H}$ : [promptly] increase. ... Like that's sort of my gut instinct. [pauses] I can actually reason out why... I can actually find reasons that it's either one.

For this student, the statistical idea of entropy was an anchoring idea that finally allowed her to resolve her confusion, at least temporarily:

$\mathrm{H}$ : Increase. In that it has the most possible number of, um, micro- or macrostates. Yeah, that makes more sense now.

Although she appeared settled, the student apparently continued to look for confirmation and over seven minutes later she returned to this prediction:

$\mathrm{H}$ : The entropy of the Universe has to be greater than or equal to zero, which goes back and reasserts my... [prior prediction of] increase.

While the statistical idea seemed to help, she admitted that several aspects of the statistical formulation remained challenging to her:

$\mathrm{H}$ : In class, I really struggled with, being comfortable with, the definition of, application of, microstates versus macrostates.

Aside from moving toward that most stable equilibrium point, I can't really apply the multiplicities. 
Each of these three students (Calliope, Gladius, and Hecate) made reference to multiple resources at different points in the interview, and none appeared to have a completely correct understanding of all aspects of entropy. While they were the most dramatic examples of use of multiple resources, all of the students in the sample referred to multiple resources.

\section{Some thoughts on hidden and suppressed resources}

Subsequent to the interviews, we wondered at the fact that the association of entropy and order in equilibrium has not appeared in other contexts. We have posed a number of written questions in order to probe further this connection between entropy and order or disorder. Very few students spontaneously gave explanations associating order with an increase in entropy. However, it is clear even within the interviews that students respond to cues from instructors and take up language even if does not represent their initial thinking. The best example comes from the responses of Gladius, and her shifting language when talking about order; it seems in retrospect that the interviewer, despite listening very carefully to student language, did not recognize the potential significance of Gladius referring to "that would be disorder" during the interview. Later Gladius was referring to "disordered" or "more disorder," but it is not clear whether this is her preferred language or represents her adoption of the interviewer's language. Written problems do not allow much latitude for students to use nonstandard language, and instructors in class might not always notice nuances in student phrasing, or may correct the student language without attending to the substance of the reasoning behind it. Subsequent to these interviews, the instructor noticed at least one student using almost this same phrasing in class, referring to equilibrium as a more ordered state.

\section{DISCUSSION}

The student responses to these interview prompts suggest that student thinking about entropy is more complex than previous research might have indicated. Most of the students in our study did not employ a small number of strongly held ideas, but rather shifted frequently among a larger number of ideas, most of which were at least potentially productive.

Among the ideas employed by students, those that had been introduced by the course instructor were not the most prevalent. Association of entropy with multiplicity was uncommon, and even students who expressed a preference for this idea had difficulty in using it to make certain predictions. None of the students explicitly invoked the Clausius algorithm for macroscopic entropy changes, despite its emphasis in the course.

On the other hand, associations that had not been encouraged by the instructor were quite prevalent. Some of the responses mirrored previously published results. Many students used conservation resources for entropy problems, despite the fact that entropy is not generally conserved. Many students used the notion of entropy as a measure of disorder despite it leading to confusion on certain predictions. In particular, students struggled to reconcile the notion of entropy as disorder with an intuition that thermal equilibrium is a natural, and thus ordered, state. Students in this category either predicted that the entropy of a system would decrease as the system approached thermal equilibrium or shifted back and forth between predictions.

The data suggest that efforts by instructors to banish the idea of entropy as a measure of disorder may have only limited success. Students were not exposed to this idea in this course and yet many of the students in interviews employed it repeatedly. Students used a variety of other metaphors not explicitly introduced in the class, connecting entropy with macroscopic or particle-level disorganization or dispersal. Whether students are encountering these ideas in formal classroom settings or in popular depictions of entropy as disorganization cannot be determined from our data, but the association of entropy with order and disorganization are clearly widespread despite efforts to the contrary.

While this work was not directly intended to improve instruction, we believe that a better characterization of student thinking about entropy will help practitioners as well as researchers.

\section{ACKNOWLEDGMENTS}

We thank our colleagues John Thompson and Don Mountcastle at the University of Maine, along with former students Trevor Smith and Brandon Bucy; David Meltzer at Arizona State University; and Warren Christensen at North Dakota State. We further thank colleagues at the CSUF Catalyst Center for reading an early draft of this manuscript, particularly Sissi Li and Mary Emenike. The work was supported by the National Science Foundation Grant No. DUE-0817335. 
[1] S. Kesidou and R. Duit, Students' conceptions of the second law of thermodynamics-an interpretive study, J. Res. Sci. Teach. 30, 85 (1988).

[2] B. R. Bucy, Ph.D. thesis, University of Maine, 2007.

[3] T. G. Amin, F. Jeppsson, J. Haglund, and H. Strömdahl, Arrow of time: Metaphorical construals of entropy and the second law of thermodynamics, Sci. Educ. 96, 818 (2012); C. Brosseau and J. Viard, 'Quelques réflexions sur le concept d'entropie issues d'un enseignement de thermodynamique‘, Enseñanza de las Cientias 10, 13 (1992).

[4] M. J. Cochran and P. R. L. Heron, Development and assessment of research-based tutorials on heat engines and the second law of thermodynamics, Am. J. Phys. 74, 734 (2006).

[5] A. R. Daane, S. Vokos, and R. E. Scherr, Conserving energy in physics and society: Creating an integrated model of energy and the second law of thermodynamics, AIP Conf. Proc. 1513, 114 (2013).

[6] B. D. Geller, B. W. Dreyfus, J. Gouvea, V. Sawtelle, C. Turpen, and E. F. Redish, Entropy and spontaneity in an introductory physics course for life science students, Am. J. Phys. 82, 394 (2014).

[7] W. M. Christensen, D. E. Meltzer, and C. A. Ogilvie, Student ideas regarding entropy and the second law of thermodynamics in an introductory physics course, Am. J. Phys. 77, 907 (2009).

[8] E. Langbeheim, S. A. Safran, S. Livne, and E. Yerushalmi, Evolution in students' understanding of thermal physics with increasing complexity, Phys. Rev. ST Phys. Educ. Res. 9, 020117 (2013).

[9] D. Styer, Insight into entropy, Am. J. Phys. 68, 1090 (2000).

[10] F. L. Lambert, Disorder - A Cracked Crutch for Supporting Entropy Discussions, J. Chem. Educ. 79, 187 (2002).

[11] H. S. Leff, Removing the mystery of entropy and thermodynamics. Part I, Phys. Teach. 50, 28 (2012); Removing the mystery of entropy and thermodynamics. Part II, Phys.
Teach. 50, 87 (2012); Removing the mystery of entropy and thermodynamics. Part III, Phys. Teach. 50, 170 (2012); Removing the mystery of entropy and thermodynamics. Part IV, Phys. Teach. 50, 215 (2012); Removing the mystery of entropy and thermodynamics. Part V, Phys. Teach. 50, 274 (2012).

[12] T. Moore and D. V. Schroeder, A different approach to introducing statistical mechanics, Am. J. Phys. 65, 26 (1997).

[13] T. A. Moore, Six Ideas That Shaped Physics (McGrawHill, New York, 2003).

[14] D. V. Schroeder, An Introduction to Thermal Physics (Addison-Wesley, San Francisco, 2000).

[15] P. R. L. Heron, in Empirical Investigations of Learning, and Teaching, Part I: Examining, and Interpreting Student Thinking, Proceedings of the "Enrico Fermi" Summer School on Physics Education Research, edited by E. F. Redish and M. Vicentini (Italian Physical Society, Varenna, Italy, 2003), pp. 341-351.

[16] D. Hammer, Student resources for learning introductory physics, Am. J. Phys. 68, S52 (2000).

[17] M. E. Loverude, Student understanding of basic probability concepts in an upper-division thermal physics course, AIP Conf. Proc. 1179, 189 (2009); Investigating student understanding for a statistical analysis of two thermally interacting solids, AIP Conf. Proc. 1289, 213 (2010); (unpublished).

[18] A. A. diSessa, Towards an epistemology of physics, Cognit. Instr. 10, 105 (1993).

[19] J. Minstrell, in Proceedings of the Conference on Research in Physics Learning (IPN, Kiel, Germany, 1992), pp. 110-128.

[20] M. Loverude, Research and curriculum development on student understanding of the microcanonical ensemble, Part I: Counting microstates [Phys. Rev. ST Phys. Educ. Res (to be published)].

[21] In the courses students were taught F, G, and $\mathrm{H}$. 